whose behaviour, through being sufficiently deviant, poses a risk to others.

For a planned intervention to be effective, the people in these groups, or their carers, will need sufficient information to understand the need for, and to agree to, any action. The challenge for professionals is to communicate the process of implementation of specially targeted interventions designed to reduce the risk of injury to themselves or to others in the community.
1 Ward $\mathrm{H}$, Cave J, Morrison A, et al. Pedestrian activity and accident risk. Basingstoke: AA Foundation for Road Safety Research, 1994.

2 West RJ. Individual differences in accident risk: a review of findings and an examination of methods. Behavioura $\bar{D}$ research in road safety VI. Proceedings of a seminar at Royal Holloway College, University of London, 4-5 September 1995. PR3138/96. Crowthorne: Transpore Research Laboratory, 1996.

3 Christie N. The high risk pedestrian: socio-economic and environmental factors in their accidents. Project Reporf? (1995O 4 Wilde GJS. Target risk. Toronto: PDE Publications, 1994.

\title{
Population strategies for prevention? If only it were that simple!
}

\author{
Jerry Moller
}

The paper by Kendrick and Marsh (p170) sparked this Dissent. On the basis of this one study, in one general practice in the UK, the authors suggest that injury prevention targeted at groups at higher risk is not worthwhile and that a universal approach to injury prevention is preferable. I disagree, despite the thoughtful Opinion of Ward (p160) who defends this conclusion.

The debate about universal (or population based) versus high risk strategies has ebbed and flowed for 20 years. It was a central issue in arguments about the welfare state, ${ }^{1}$ and reappeared in suggestions that indicated that health promotion has contributed to increasing differentials between rich and poor by adopting a universal approach. Little has been written about this issue with respect to injury, but as Kendrick and Marsh point out, many recommendations have been made for targeting high risk groups, especially poor children, based on injury rate differentials. The lack of debate has created a climate where the responses of policy makers and researchers are often over simplified.

We must first consider why the leap from a single study to the conclusion that universal strategies are the best approach is simplistic, despite Ward's support. The Kendrick and Marsh paper considers only some of the layers of complexity and argues that universal approaches may increase the rich-poor differential. Targeted strategies are necessary, the authors say, if this disadvantage is to be avoided.

\section{Injury Information Services, Australian Institute of Health and Welfare, National Injury Surveillance Unit, Adelaide, South Australia}

Correspondence to: Mr J Moller, PO Box 38, Mt Compass, SA 5210, Australia.

\section{Measuring risk}

The measurement of risk is itself, a complex issue. How does the risk of individuals, or communities relate to population risk? At what level of severity should risk be measured? There may be little difference in risk measured across the full range of severity ranging from first aid to medical treatment to death, but large differentials among the most severe injuries, such as those resulting in long term disability or death. One subpopulation, indi- $\frac{\mathbb{D}}{\mathbb{Q}}$ cated by broad measures to be at higher risk, may not have a measurable difference in injury rates in a short term or cross sectional study $\overrightarrow{x 0}$ purely because of random variations in injury incidence. Differences may also be hidden by inappropriate use of age standardization or byo failure to consider maturation effects.

It should be remembered that the indicatorso of high risk are often relatively insensitive proxyळ measures. Jolly et al have shown that composite measures of socioeconomic status underesti-O mate the relationship between poverty and injury rates because components of socio economic status, such as education and income, correlate in opposite directions with injury rates under some circumstances. ${ }^{2}$

In the light of this, it is not surprising that some studies will show a strong relationship between poverty and risk while others show no such relationship. Equally, just as it is falla-? cious to believe that every individual in a high risk group has an elevated risk of injury, it is also a fallacy to believe that every under- $\frac{0}{3}$ privileged community will experience elevated injury rates.

\section{Trends in injury patterns for the population}

Cross sectional and short term studies easily lead to misunderstandings about theo need for targeted interventions. Consider figo 1: a small difference in injury rate occurs at the beginning; it increases over time, but the? rates of injury drop for both groups. The difference is insufficient to be seen to justify $a \frac{\text { O }}{\Omega}$ targeted intervention and a universal interven- $-\stackrel{\Phi}{\Omega}$ tion is commenced. It has a larger effect on $\stackrel{\mathbb{Q}}{\AA}$ the low risk group than the high risk group The differential is increased, even thougho both groups have benefited. Failure to identifyo the need to undertake a targeted intervention has resulted in an increased differential. The? difference may have become more entrenched and the cost of intervening may have increased. 


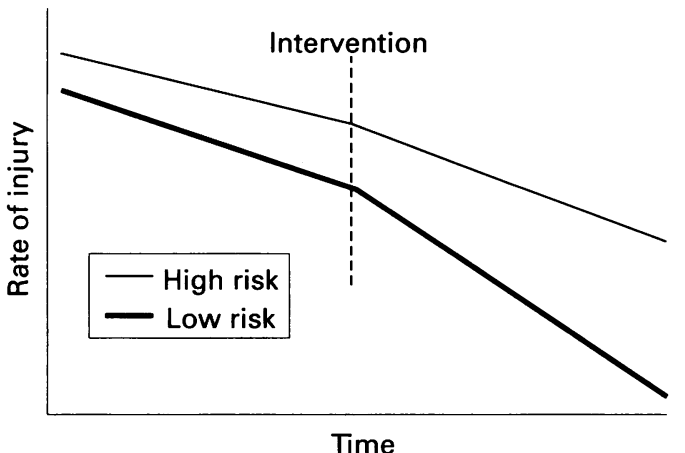

Figure 1 Variations of rate of injury over time and the differing impacts of intervention on high and low risk groups.

Value systems, economic rationalism, and social justice

The decision about whether to target interventions is not just a scientific matter. Even if it were possible to accurately measure relative risk, present evidence for effective intervention and measure the impacts, the debate about whether to target the needy would still rage. Value judgments about the worthy and nonworthy poor, old arguments about accident prone individuals and communities, debate about the best value for money, and plain old protection of established power, have much more influence on what is done than science ever will. The current ascendancy of economic rationalism will ensure that the maximum number of injuries will be prevented at the lowest possible cost. This means that the groups easiest to change and able to reach by mass strategies will receive the greatest attention. Those at lower risk are a much better economic bet than those at high risk because, in developed societies, there are many more of them and only marginal changes in rates will result in the greatest numbers of injuries being prevented. Equally, the well off and well educated are the easiest to reach and the most likely to be affected by behaviour change

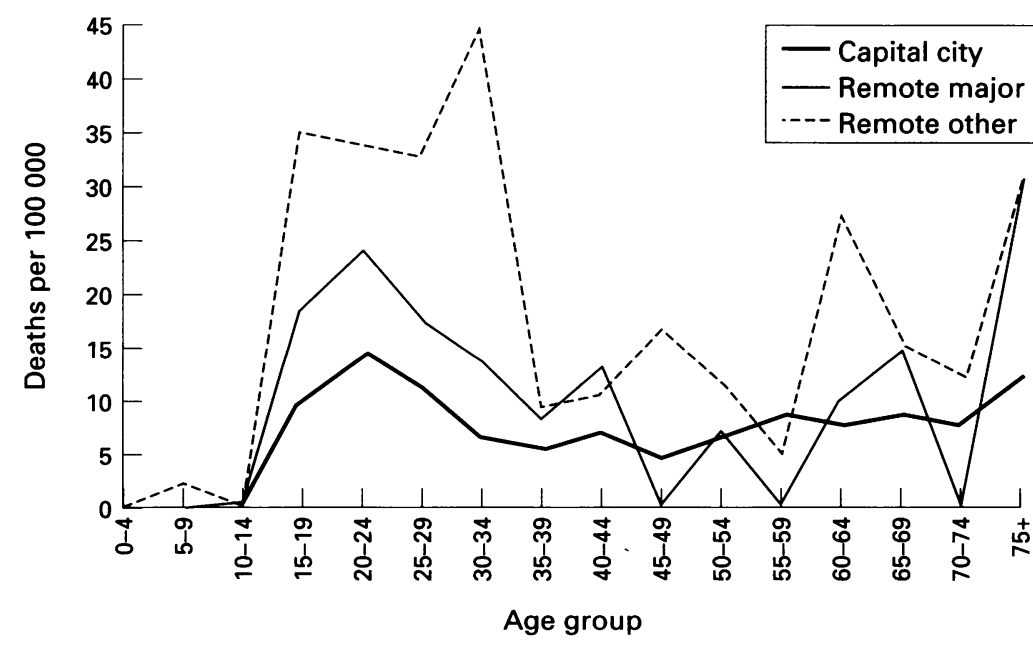

Figure 2 Annual average male motor vehicle driver death rates: 'remote major' and 'remote other' areas compared with 'capital city' by age, Australia 1990-2. methods. Those who are well off are also likely to be in a position to influence the targeting of environmental changes by political influence.

Principles of social justice and equity are less fashionable now than during the 1970s and 1980s. This decade has seen increasing differentials emerging between the rich and the poor and between rich and poor nations. The economic models applied on the developed Western world are likely to increase these differences. Any move to reduce efforts aimed at preventing injury among the high risk groups will multiply the impacts of the economic decision. Those most economically disadvantaged live in the highest risk environments, experience the highest rate of serious injury and death, and suffer the highest rates of other major diseases resulting in premature death. In these circumstances it seems better in principle to err toward targeting the poor and the needy, than to follow the more popular trend of making the rich richer and the safe safer.

\section{Effectiveness and interventions}

Little evidence has been presented about the relative effectiveness of injury interventions on different target groups. Health promotion strategies have been shown to be more effective on the educated middle and upper classes than on the poor. ${ }^{3}$ Even if the rate of injury of two groups were the same, an intervention may not impact equally on them. Marketing strategists are acutely aware that products need to be designed to appeal to different target groups. A dismissal of the need to target interventions correctly at different groups flies in the face of evidence of the need to segment target populations to achieve effectiveness and efficiency. The need for targeted interventions therefore does not rely on measured risk differential alone. It may also be justified on the basis of ensuring that appropriate methods are available for subsegments of the population.

\section{Effects of a universal strategy}

The outcome of a universal approach to injury prevention can be seen in the patterns of injury in Australia. While great successes have been achieved overall, serious problems have developed and remain entrenched for some groups.

\section{Differentials in road safety}

Australia has been a leader in road safety. It has achieved huge reductions in road related deaths and crash casualties. The strategies have been focused on reducing overall victim numbers. The reductions have largely been achieved for urban dwellers and very high differentials in road related death remain. Rural and remote dwellers exhibit death rates of up to 2.7 times their city counterparts (see fig 2 for male rates). ${ }^{4}$ Equally young male rates in both city and country remain much higher than rates for females and other age groups. Little is known about the socioeconomic status of drivers or victims, although spatial mapping of road related 
Rate ratios of death and hospitalisation comparing indigenous Australians and other Australians 1991-2

\begin{tabular}{lccc}
\hline & & \multicolumn{2}{c}{ Hospitalisations } \\
\cline { 3 - 4 } Cause & Deaths & Males & Females \\
\hline Transport & 3.4 & 1.7 & 1.3 \\
Drowning & 4.8 & 0.7 & 1.9 \\
Poisoning medication & 2.2 & 1.7 & 2.1 \\
Poisoning other & 17.5 & 2.0 & 2.1 \\
Falls & 1.2 & 2.2 & 1.7 \\
Fire and flames & 10.5 & 5.1 & 4.7 \\
Self harm & 3.4 & 2.3 & 2.3 \\
Interpersonal violence & 10.8 & 10.9 & 46.0 \\
\hline
\end{tabular}

deaths by place of usual residence shows higher age standardised mortality rates in areas with high concentrations of poorly educated and lower income residents. ${ }^{5}$

A decision to apply a universal model to prevention is often accompanied by a lack of rigour in identifying high risk groups. The use of the road toll as the key indicator of progress or otherwise reinforces a universal approach and has resulted in increasing differentials. This leads to the existence of entrenched risk and it is only recently that Australian road safety policy makers have started to examine how they can tackle problems of rural crashes, young male injury and death, and chronic alcohol users. The targeting of the poor is not yet on the agenda.

\section{Indigenous injury}

Indigenous Australians also experience a very high rate of injury. Over the past two years the extent of this problem has been identified more accurately. ${ }^{67}$ Table 1 shows a summary of rate ratios comparing injuries among indigenous and non-indigenous Australians. It is feasible that a cross sectional study in one local area would not replicate these figures and show little difference. On the other hand, a recent and as yet unpublished study in a small indigenous community in remote Australia has shown injury rates much higher than the national average. It has also demonstrated that national data do not accurately collect information from the areas studied.

\section{Conclusions}

If only life were more simple. If only risk groups could be identified and risks measured $\overline{5}$ without error. If only science ruled the world and value judgments did not have to be made. $\vec{\Phi}$ This view is called the naturalistic fallacy. It is logically impossible to decide what ought to be done on the basis of fact without making valueo judgments. The stark reality is that not only is $\underline{\underline{O}}$ it logically impossible, but it doesn't happen in real life. Currently, value systems and policyథ direction are leading to increased disadvantage. Those who are doing best, are fighting tomaintain that status, and those who are disadvantaged are becoming increasingly so.

A universal approach to injury prevention? will only exacerbate the problems of the $\dot{\omega}$ disadvantaged. Targeted programs are neces- $\omega$ sary to ensure those health differentials be- $\vec{N}$ tween the rich and the poor do not increase. 0 Even when a study shows in a particular? instance that the differential cannot be demon-cs strated, it is necessary to ask whether the null $\mathbb{D}^{\circ}$ result is due to targeted intervention already in place. It is also essential to assess whether ao differential will be created by the differing $\stackrel{\mathbb{T}}{-}$ impact of universal interventions on the $\vec{\theta}$ advantaged and disadvantaged. While univer-. sal interventions have their place, we have noto yet reached a stage where we can abandon our commitment to interventions targeted to high risk groups.

1 Wilensky H, Wilensky LC, Harold L. Industrial society and $\overline{\bar{O}}$ social welfare: the impact of industrialisation on the supply and organisation of social welfare services in the United Stare New York: Free Press, 1965.

2 Jolly D, Moller J, Volkmer R. The socioeconomic context of child injury in Australia. $\mathcal{f}$ Paediatr Child Health 1993; 29: 438-44.

3 Blaxter M. Health and lifestyles. London, New York: Tavi stock/Routledge, 1990

4 Moller J. The spatial distribution of injury deaths in Australia. Australian injury prevention bulletin issue 8. (1) Adelaide: National Injury Surveillance Unit, 1995.

5 Moller J. An atlas of injury death in Australia 1990-1992. 음 Adelaide: National Injury Surveillance Unit, 1995

6 Harrison J, Moller J. Injury mortality amongst Aboriginal Australians. Australian injury prevention bulletin issue 7. Adelaide: National Injury Serveillance Unit, 1994.

7 Moller J, Dolinis J, Cripps R. Aboriginal and Torres Strait islander peoples injury related hospitalisations. Adelaide: National Injury Surveillance Unit, 1996. 